DOI: 10.2478/awutp-2019-0002

\title{
GERMANIUM GRADIENT OPTIMIZATION FOR HIGH-SPEED SILICON GERMANIUM HETERO-JUNCTION BIPOLAR TRANSISTORS
}

\author{
Abdelkader Khadir ${ }^{1,2, *}$, Nouredine Sengouga ${ }^{1}$, Mohamed Kamel Abdelhafidi ${ }^{1,2}$ \\ ${ }^{1}$ Laboratory of metallic and semiconducting materials, University of Biskra, Biskra, Algeria \\ ${ }^{2}$ Materials Science and Informatics Laboratory, University of Djelfa, Djelfa, Algeria \\ *Corresponding author: Email: Khadir71@yahoo.fr
}

\begin{tabular}{|c|c|}
\hline Article Info & Abstract \\
\hline $\begin{array}{l}\text { Received: } 29.09 .2019 \\
\text { Accepted: } 27.11 .2019 \\
\\
\text { Keywords: SiGe HBT; } \\
\text { SILVACO; Trapezoidal } \\
\text { profile; Current Gain; } \\
\text { Cut-off frequency; } \\
\text { Maximum oscillation } \\
\text { frequency. }\end{array}$ & $\begin{array}{l}\text { The effect of germanium trapezoidal profile shape on the direct current } \\
\text { (DC) current gain }\left(\beta_{\mathrm{F}}\right) \text {, cut-off frequency }\left(f_{\mathrm{T}}\right) \text { and maximum oscillation frequency } \\
\text { ( } f_{\mathrm{MAX}} \text { ) of silicon-germanium (SiGe) hetero-junction bipolar transistors (HBTs) has } \\
\text { been investigated. The energy balance (EB), hydrodynamic (HD) and drift- } \\
\text { diffusion (DD) physical transport models in SILVACO technology computer aided } \\
\text { design (T-CAD) simulator were used. It was found that the current gain values } \\
\text { using energy balance model are higher than hydrodynamic and much higher than } \\
\text { those corresponding to drift-diffusion. Moreover, decreasing the germanium } \\
\text { gradient slope towards the collector side of the base enhances the maximum } \\
\text { oscillation frequencies using HD and EB models whilst, they remain stable for DD } \\
\text { model. }\end{array}$ \\
\hline
\end{tabular}

\section{Introduction}

Despite that silicon (Si) and germanium (Ge) combination idea for use in bipolar transistor engineering is old, it was only in 1987 where the first SiGe hetero-structure bipolar transistor (HBT) was reported [1]. In the 1990s with the revolution in bipolar transistor design, SiGe HBTs have emerged as serious contenders for analog, digital and mixed signal radio frequency (RF) application [2]. Previously, hetero-junction bipolar transistors had only been available in compound semiconductor technologies, such as AlGaAs/GaAs [2]. The formation of an effective hetero-junction requires two semiconductors with similar lattice spacing. Si and Ge have a relatively large lattice mismatch of $4.2 \%$, so it is very difficult to form a hetero-junction between $\mathrm{Si}$ and $\mathrm{SiGe}$ without misfit dislocations at the interface. Fortunately, however, in the 1980s a good hetero-junction was obtained with thin SiGe layers and low Ge content (relatively below 30\%) [3, 4]. In these cases, SiGe layer grows under 
strain so that it fits perfectly onto the silicon lattice without the generation of misfit dislocations. The vital technology breakthrough that led to the emergence of $\mathrm{SiGe}$ is the epitaxial growth of reproducible strained or pseudomorphic, SiGe layers. Nowadays, the market for SiGe HBTs has grown rapidly to satisfy the demand for applications such as wireless communications, high-data-rate wireline and automotive radar [5]. SiGe HBTs with cut-off frequencies of 300 and $505 \mathrm{GHz}$ and maximum oscillation frequencies of 500 and 720 $\mathrm{GHz}$ were demonstrated [5-8]. In this paper, we investigate the effect of Ge gradient profile in SiGe base on $\beta_{\mathrm{F}}, f_{\mathrm{T}}$, and $f_{\mathrm{MAX}}$ of SiGe HBTs using the drift-diffusion, hydrodynamic and energy balance models implanted in the two-dimensional (2D) Atlas module from SILVACO T-CAD. The SiGe HBT structure is based on the state-of-the-art SiGe HBT technology [5, 6]. In section 2, the structure and physical models used in this work are described. The results with analysis are discussed in section 4. Finally, a conclusion is given in the last section.

\section{Modeling and simulation}

The drift-diffusion model is the simplest model for describing charge transport in semiconductor devices [9]; it neglects non-local transport effects. In some cases such low dimensional structures, the DD model is not sufficient. More suitable models have to be used such as energy balance and hydrodynamic. The energy balance transport model (EBTM) follows the derivation by Stratton from the Boltzmann transport equation $[10,11,12]$. With some assumptions, it will decompose into the hydrodynamic model [13, 14, 15].

\subsection{Device structure}

In this work, a comparison of three SiGe HBTs is carried out using three different germanium trapezoidal profile shapes: profile 1 (HBT1), profile 2 (HBT2) and profile 3 (HBT3). Figure 1 shows the geometry of the studied SiGe HBT. All perimeters are the same for the devices and the only difference resides in the base germanium profile. The total emitter area is assumed to be $0.12 \times 0.96 \mu \mathrm{m}^{2}$ [5]. 


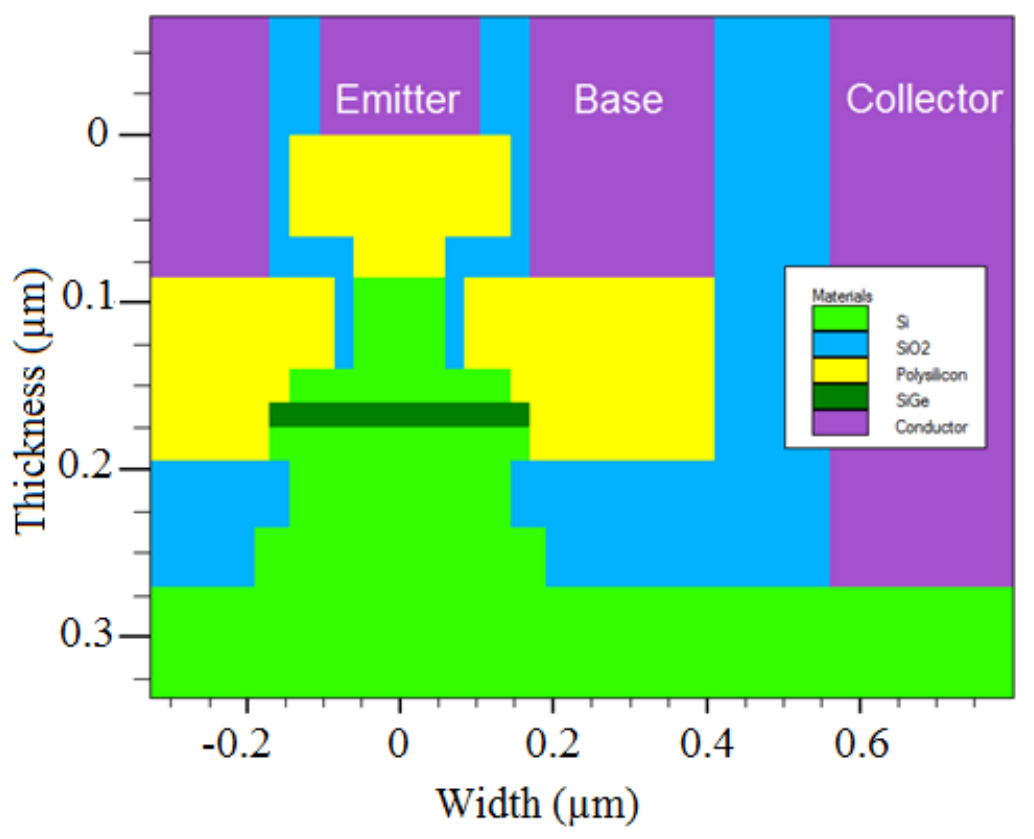

Fig. 1. SiGe HBT simulated structure.

The germanium profile in the base and vertical one-dimension (1D) doping profile are presented in Figure 2. The germanium peak composition equals to 0.28. In this figure, the net doping concentration in the three regions of the SiGe HBT; emitter, base, and collector (left axis) is presented with bleu solid line. The emitter region is high n-doped with a uniform concentration which falls from $10^{21} \mathrm{~cm}^{-3}\left(1 / \mathrm{cm}^{3}\right)$ near the emitter contact to $2 \times 10^{18} \mathrm{~cm}^{-3}$ $\left(1 / \mathrm{cm}^{3}\right)$ near the base region $\left(\mathrm{cm}^{-3}\right.$ means dopant concentration per $\mathrm{cm}^{3)}$. The base is $\mathrm{p}$-doped with a Gaussian doping profile of a peak concentration of $8 \times 10^{19} \mathrm{~cm}^{-3}$ which is inspired from [16]. The collector doping is low near the base side and is being increased gradually to be high in the collector bulk and near its contact to reduce the collector resistance. From its side, the germanium composition in the base is presented with dashed lines for the three devices (right axis). The germanium composition in the base varies according to $x$ ratio in $\mathrm{Si}_{1-x} \mathrm{Ge}_{x}$; when $x=0$ we get silicon and when $x=1$ we get germanium. In our case $x$ varies from about 0.25 at the emitter side of the base to 0.28 at the collector side of the base with three different shapes (trapezoidal profile).

The parameters used in this simulation such as doping, band-gap narrowing, low-field mobility, effective lifetime, germanium band-gap narrowing and others are taken from [10, 17]. During all simulations, material parameters, physical models and structure dimensions and perimeters are being kept unchanged. 


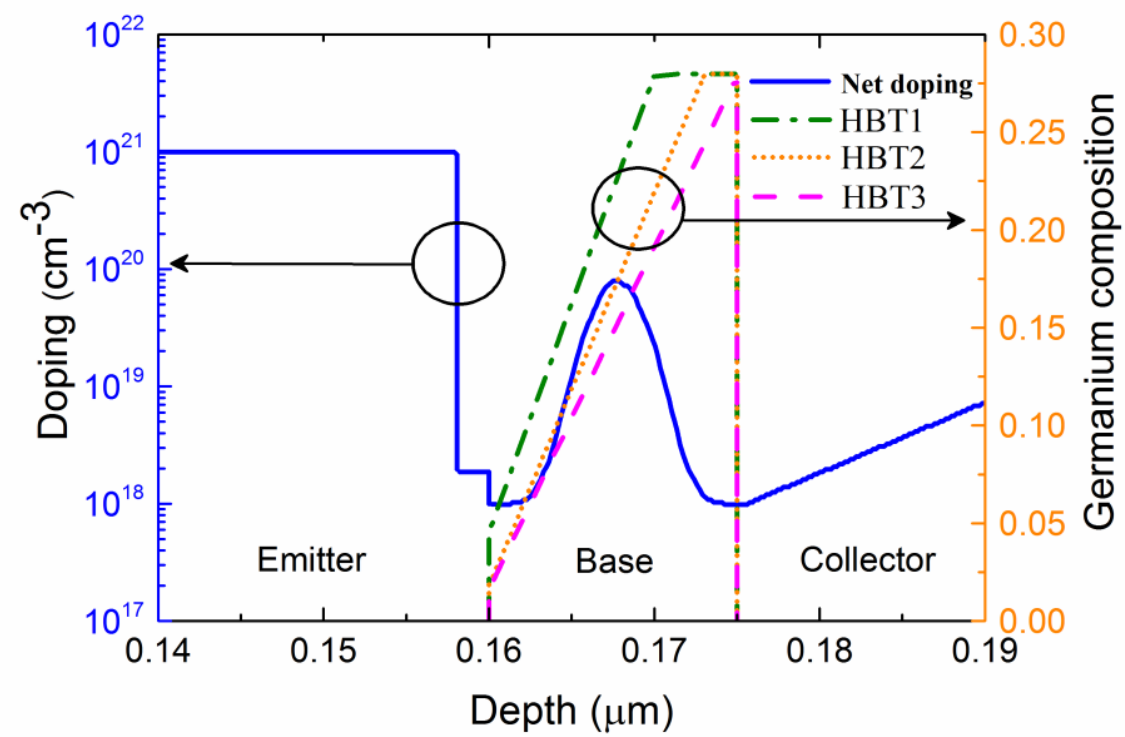

Fig. 2. Germanium profile shapes and doping used in the simulation [16]

\subsection{Physical parameters}

Advances in silicon and $\mathrm{Si}_{1-x} \mathrm{Ge}_{x}$ alloys growth have allowed the potential for using bandgap engineering to construct hetero-junction devices such as HBTs and HEMTs using these materials.

The following section describes the functional relationship between Ge mole fraction $x$, and $\mathrm{SiGe}$ material characteristics necessary for device simulation.

\section{2.1. Bandgap}

Bandgap is one of the most fundamental parameters for any material. For SiGe, the dependence of bandgap on Ge mole fraction, $x$, is divided into ranges as follows $[10,18]$ :

$$
\begin{gathered}
\text { For } \quad x \leq 0,245 \\
\mathrm{E}_{\mathrm{g}}=1.08+(x \times(0.945-1.08)) / 0.245 \\
\text { For } \quad 0.245 \leq x \leq 0.35 \\
\mathrm{E}_{\mathrm{g}}=0.945+(x-0.245) \times(0.87-0.945) /(0.35-0.245)
\end{gathered}
$$

Temperature dependence of the bandgap of SiGe is calculated the same as for silicon 
except that $E_{g \alpha}$ and $E_{g \beta}$ are a function of $G e$ mole fraction $x$ as follows:

$$
\mathrm{E}_{\mathrm{g}}\left(\mathrm{T}_{\mathrm{L}}\right)=E_{g}+E_{g \alpha}\left[\frac{300^{2}}{300+E_{g \beta}}-\frac{T_{L}^{2}}{T_{L}+E_{g \beta}}\right]
$$

Where:

$$
\begin{gathered}
E_{g \alpha}=(4.73+x \times(4.77-4.73)) \times 10^{-4} \\
E_{g \beta}=636+x \times(235-636)
\end{gathered}
$$

\subsubsection{Electron Affinity}

The electron affinity of SiGe is taken to be constant (4.17) with respect to composition.

\subsubsection{Density of States}

The density of states for SiGe depends upon Ge mole fraction, $x$ composition, according to :

$$
\begin{aligned}
& N_{C}=2.8 \times 10^{19}+x \times\left(1.04 \times 10^{19}-2.8 \times 10^{19}\right) \\
& N_{V}=1.04 \times 10^{19}+x \times\left(6.0 \times 10^{18}-1.04 \times 10^{19}\right)
\end{aligned}
$$

\subsubsection{Dielectric Function}

Compositional dependence of static dielectric constant of SiGe is given by:

$$
\varepsilon=11.8+4.2 \times x
$$

\subsubsection{Low Field Mobility}

SiGe low field electron and hole mobilities at room temperature are given by [17] as:

$$
\begin{array}{ll}
\mu_{n} \approx(1396-4315 \times x)_{c m^{2} V^{-1} S^{-1}} & 0 \leq x \leq 0.3 \text { at } 300 \mathrm{~K} \\
\mu_{P} \approx(450-865 \times x)_{c m^{2} V^{-1} S^{-1}} & 0 \leq \mathrm{x} \leq 0.3 \text { at } 300 \mathrm{~K}
\end{array}
$$

\subsubsection{Velocity Saturation}

In SiGe, the temperature dependent velocity saturation, used in field dependent mobility model is defined by the following equations.

$$
\begin{aligned}
& V_{S A T N}=1.38 \times 10^{7} \cdot \sqrt{\tanh \left(\frac{175}{T_{L}}\right)} \\
& V_{S A T P}=9.05 \times 10^{6} \cdot \sqrt{\tanh \left(\frac{312}{T_{L}}\right)}
\end{aligned}
$$

Where $T_{L}$ denotes the lattice temperature.

\section{Results and discussions}

The effects of germanium trapezoidal profile shapes on the DC current gain, the cut- 
off frequency and the maximum oscillation frequency using drift diffusion, hydrodynamic and energy balance models are performed by the use of Atlas module from SILVACO TCAD. The electric parameters results from this simulation are presented and compared. The DC current gain versus base-emitter voltage of the three HBTs is shown in Figure 3. We notice that HBT1, HBT2 and HBT3 have a maximum current gain of 717, 705 and 680 using EB model, respectively. The change of the germanium profile shape affects slightly the DC current gain; the gain decreases about 8\% for DD and 5\% for HD and EB transport models from HBT1 to HBT3. The change of the trapezoidal profile from profile1 to profile 3 induces a small reduction of the collector current, base current and the current gain, too and this agrees with theory, which indicates that the gain enhancement varies exponentially with the germanium composition at the emitter end of the base, whereas it varies linearly with the grading [19]. The values of $\beta_{\mathrm{F}}$ using EB model are higher than HD (about 1.67 times) and much higher than DD (about 3.5 times). The experimental results that are used for comparison in our work are taken from [5]. In these experimental results, the currents are normalized to an effective emitter width of $0.12 \mu \mathrm{m}$ which is the case in our device giving a peak current gain of 700 reached at $\mathrm{V}_{\mathrm{CB}}=0 \mathrm{~V}$, room temperature, and at a base-emitter voltage of $0.8 \mathrm{~V}$. This peak current gain is close to our results in the case of using energy balance transport model. Figure 3 shows clearly that the EB current gain values are so close to those obtained experimentally used for comparison (specifically for HBT1) which means that EB transport model is more accurate than HD and DD models. This difference in current gain values between the models is due to temperature effects that could give an explanation between the transport models differences.

Figure 4 shows the overlay of the gummel plots of the three devices using EB simulation alongside with experimental results from [5]. Experimental collector and base current plots are normalized to an emitter width of $0.12 \mu \mathrm{m}$ at $\mathrm{V}_{\mathrm{CB}}=0 \mathrm{~V}$ and at room temperature as the same as of our work. HBT1 gummel plots exhibits an excellent characteristics for both collector and base currents. We notice that both $I_{C}$ and $I_{B}$ currents match well experimental results (particularly HBT1) until a base-emitter voltage of $0.8 \mathrm{~V}$ after which they undergo a slight decrease. These results confirm that EB transport model is more accurate than other models. 


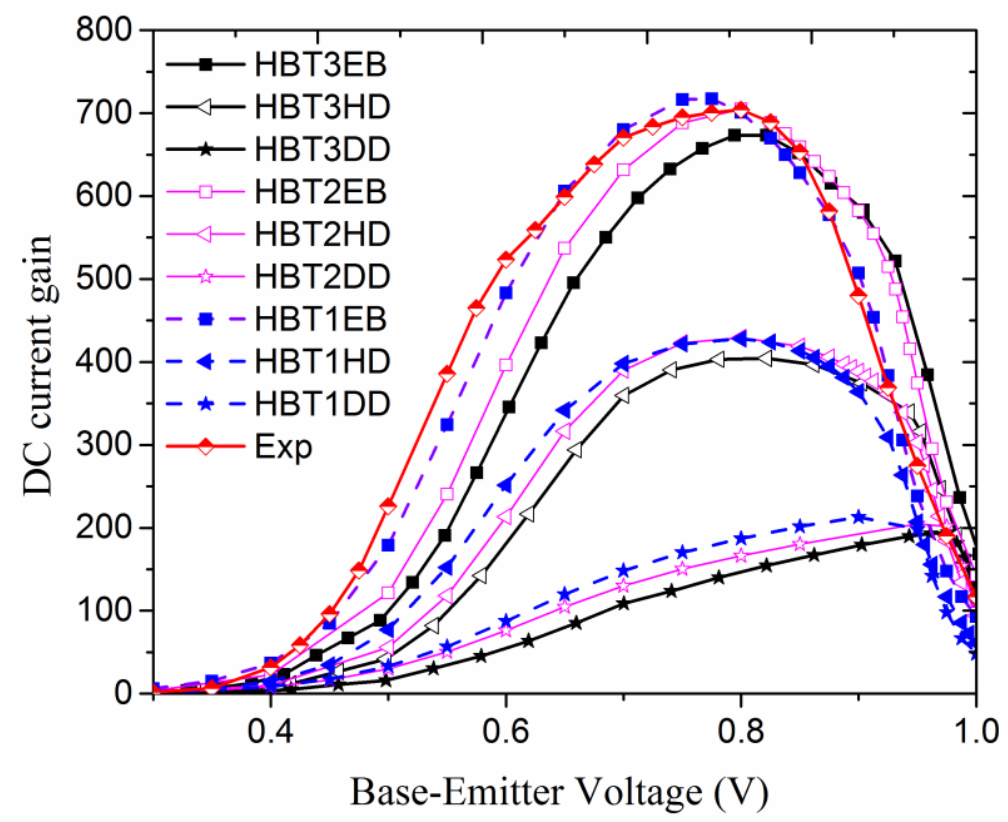

Fig. 3. DC current gain versus base-emitter voltage. Half up diamond are for experimental results from [5].

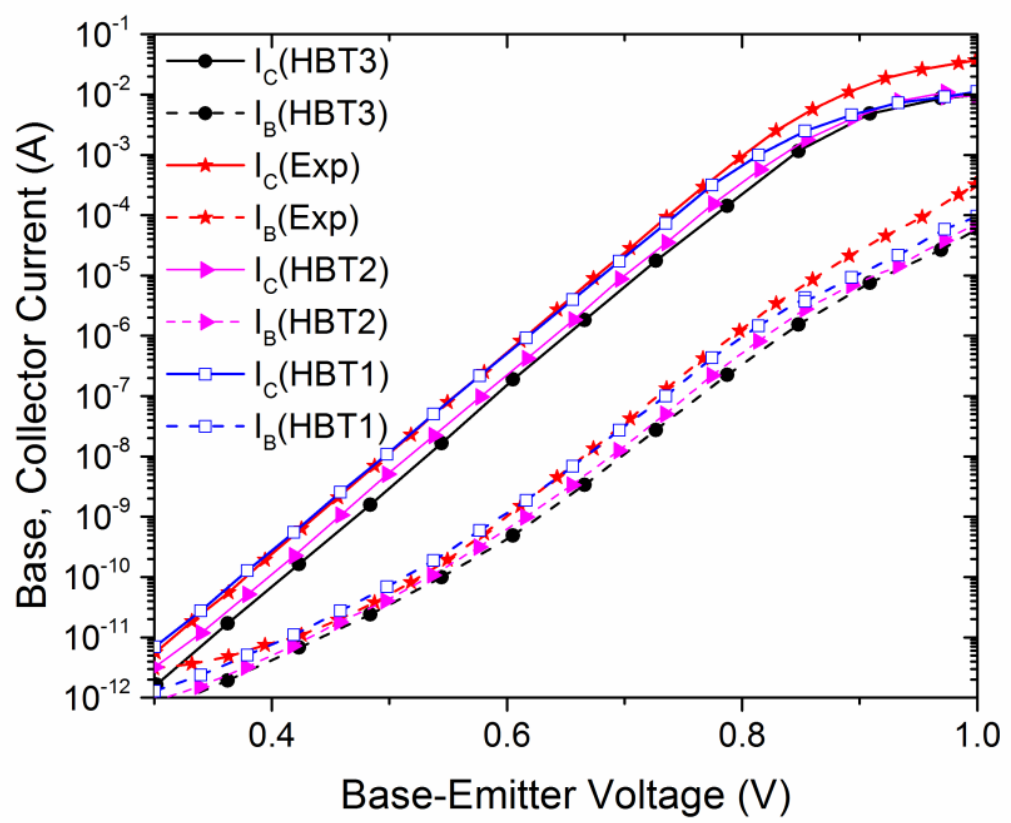

Fig. 4. EB simulation gummel plots. Stars are for experimental results [5]. 


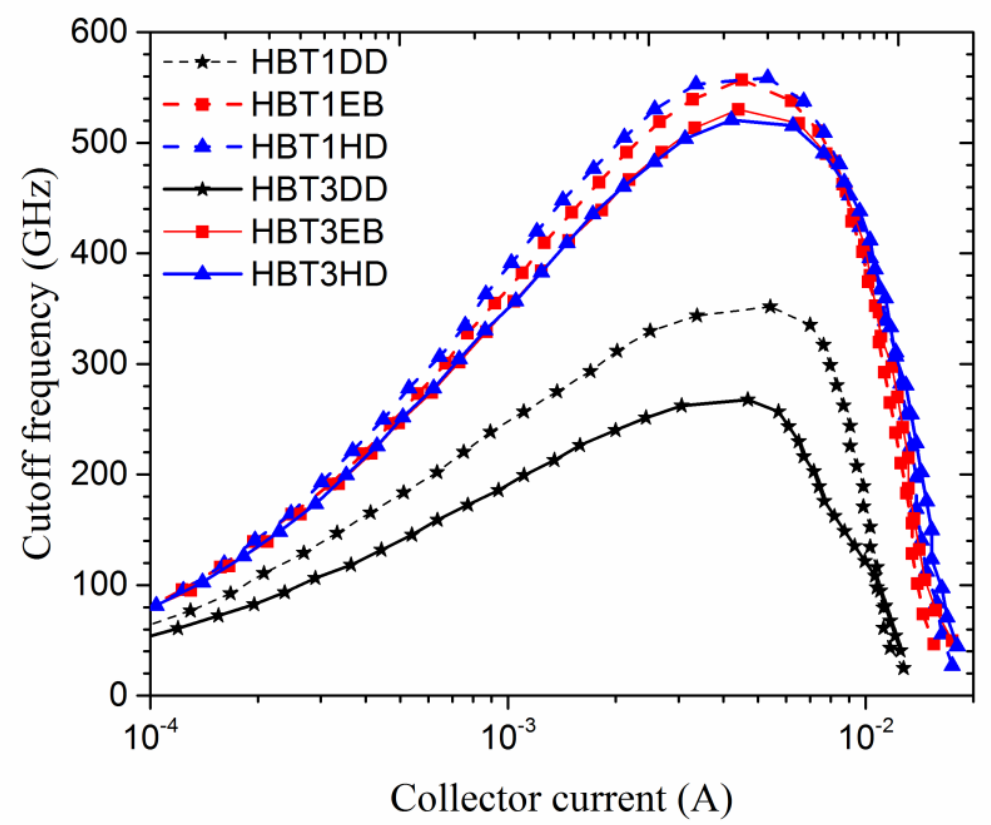

Fig. 5. The calculated cut-off frequency $f_{\mathrm{T}}$ for devices HBT1and HBT3.

The cut-off frequency $f_{\mathrm{T}}$ was first calculated according to $f_{\mathrm{T}}=\mathrm{g}_{\mathrm{cb}} /\left(6.28 \times \mathrm{C}_{\mathrm{bb}}\right)$ as shown in Figure 5, then extracted directly from the ac current gain. The maximum oscillation frequency $f_{\text {MAX }}$ was obtained from the unilateral power gain $U$, as shown in Figure 6. Table 1 recapitulates and compares the electrical parameters of the three devices under load.

Table 1. Results of $\beta_{F}, f_{T}$ and $f_{M A X}$ for devices HBT1, HBT2 and HBT3.

\begin{tabular}{|l|l|l|l|l|l|l|l|l|l|}
\cline { 2 - 10 } \multicolumn{2}{|l}{} & \multicolumn{2}{|l}{ DD } & \multicolumn{2}{l}{ HD } & \multicolumn{2}{l|}{ EB } \\
\cline { 2 - 11 } & HBT1 & HBT2 & HBT3 & HBT1 & HBT2 & HBT3 & HBT1 & HBT2 & HBT3 \\
\hline$\beta_{\mathrm{F}}$ & 212.7 & 204.8 & 194.8 & 427.8 & 429.4 & 407.2 & 717.1 & 705 & 680.7 \\
\hline $\begin{array}{l}f_{\mathrm{T}}(\mathrm{GHz}): \\
\text { calculated }\end{array}$ & 354 & 311 & 268 & 566 & 538 & 527 & 559 & 547 & 531 \\
\hline $\begin{array}{l}f_{\mathrm{T}}(\mathrm{GHz}): \\
\text { from ac } \\
\text { current gain }\end{array}$ & 322 & 314 & 268 & 432 & 563 & 612 & 263 & 442 & 521 \\
\hline $\begin{array}{l}f_{\text {MAX }}(\mathrm{GHz}): \\
\text { from U }\end{array}$ & 312 & 314 & 312 & 304 & 411 & 434 & 163 & 312 & 361 \\
\hline
\end{tabular}


From these values in Table 1, we can notice that the calculated and extracted cut-off frequencies using DD transport model are close to each other and decrease from HBT1 to HBT3. $f_{\text {MAX }}$ remains the same for the three devices. On the other hand, the calculated cut-off frequency decreases slightly using HD and EB models from HBT1 to HBT3. The cut-off and the maximum oscillation frequencies extracted directly from the ac current gain and unilateral power gain, respectively increase from HBT1 to HBT3 using HD and EB transport models. This increase in figures of merit could be explained by that the reduction in the germanium concentration at the emitter side and the shifting of the germanium peak towards the basecollector junction has as consequence a decrease in the transit time leading to an enhancement in the frequencies, particularly in the maximum oscillation frequency $f_{\text {MAX }}[19]$.

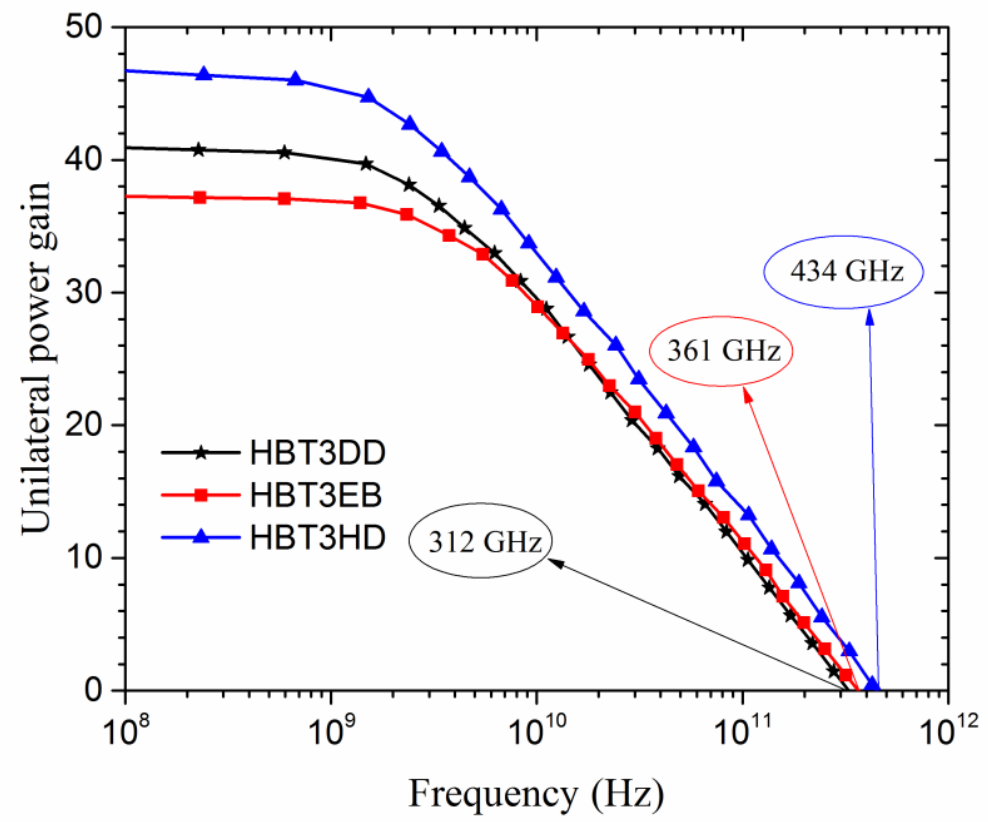

Fig. 6. Unilateral power gain against frequency to determine $f_{\text {MAX }}$ for device HBT3

\section{Conclusion}

In this work, the effects of the change of the trapezoidal profile shape on the performance of the SiGe HBT are studied (especially on the DC current gain $\beta_{\mathrm{F}}$, the cut-off frequency $f_{\mathrm{T}}$ and the maximum oscillation frequency $f_{\mathrm{MAX}}$ ). A two-dimensional energy balance (EB), hydrodynamic (HD) and drift-diffusion (DD) carriers transport models were used in the simulation. It was found that the values of the current gain using EB model are higher than the HD (about 1.7 times) and much higher than the DD (more than 3.5 times) and these EB values are in agreement with experimental. For DD transport model, the $f_{\mathrm{T}}$ 
calculated and $f_{\mathrm{T}}$ extracted from the ac current gain are quite the same and decrease from HBT1 to HBT3. $f_{\text {MAX }}$ remains the same for the three devices. The calculated cut-off frequency decreases using HD model from HBT1 to HBT3 and shows no significant change using EB model. $f_{\mathrm{T}}$ and $f_{\mathrm{MAX}}$ extracted directly from the ac current gain and unilateral power gain, respectively augment from HBT1 to HBT3 using HD and EB transport models. The results of the three transport models show remarkable differences. The phenomenon leading to these differences must be determined to keep using device simulation to develop compact models leading to understand the physical effects that occurs in a device.

\section{Acknowledgements}

The authors Acknowledge colleagues and individuals for their help and support for achieving this work.

\section{References}

[1] C. K. Maiti, G. A. Armstrong. Applications of Silicon-Germanium Hetero-structure Devices, IOP Publishing Ltd, Bristol, (2001)

[2] B. Senapati, C. K. Maiti, N. B. Chakrabati. Silicon Hetero-structure Devices for RF Wireless Communication, In Proceedings of 13th International Conference on VLSI Design (2005) 488-491

[3] A. Schuppen, A. Gruhle, H. Kibbel, U. Konig. Mesa and planar SiGe HBTs on MBE wafers, Journal of materials science: materials in electronics 6 (1995) 298-305

[4] J. W. Matthews, A. E. Blakeslee. Defects in epitaxial multilayers: III. Preparation of almost perfect multilayers, Journal of Crystal Growth 32 (1976) 265-273

[5] H. Rücker, B. Heinemann, A. Fox. Half-terahertz SiGe BiCMOS technology, In IEEE 12th Topical Meeting on Silicon Monolithic Integrated Circuits in RF Systems (2012) $133-136$

[6] B. Heinemann, et al. SiGe HBT technology with $f_{\mathrm{T}} / f_{\mathrm{MAX}}$ of $300 / 500 \mathrm{GHz}$ and $2.0 \mathrm{ps}$ CML gate delay, In IEEE 2010 International Electron Devices Meeting (2010) 688-691

[7] P. Chevalier, T.F. Meister, B. Heinemann, et al. Towards THz SiGe HBTs, In IEEE Bipolar/BiCMOS Circuits and Technology Meeting (2011) 57-65

[8] B. Heinemann, H. Rücker, R. Barth, et al. SiGe HBT with $f_{\mathrm{T}} / f_{\mathrm{MAX}}$ of $505 \mathrm{GHz} / 720$ GHz," In IEEE International Electron Devices Meeting (2016) 311-314 
[9] S. M. Sze. Semiconductor Devices: Physics and Technology, 2nd Edition, USA, (1985)

[10] Atlas user's manual, https://dynamic.silvaco.com/dynamicweb/silen/, 2012 (Accessed 30 January 2012)

[11] R. Stratton. Diffusion of hot and cold electrons in semiconductor barriers, Physical Review 126 (1962) 2002

[12] R. Stratton. Semiconductor current-flow equations diffusion and degeneracy, IEEE Transaction on Electron Devices 19 (1972) 1288-1292

[13] B. Meinerzhagen, K. Bach, I. Bozk, W. L. Eugl. A New Highly Efficient Nonlinear Relaxation Scheme for Hydrodynamic MOS Simulations, In: NUPAD IV. Workshop on Numerical Modeling of Processes and Devices for Integrated Circuits (1992) 91-96

[14] Y. Apanovich, P. Blakey, R. Cottle, E. Lyumkis, B. Polsky, A. Shur, A. Tcherniaev. Numerical simulation of sub-micrometer devices including coupled nonlocal transport and non-isothermal effects, IEEE Transactions on Electron Devices 42 (1995) 890898

[15] Y. Apanovich, E. Lyumkis, B. Polsky, A. Shur, P. Blakey. Steady-state and transiet analysis of submicron devices using energy balance and simplified hydrodynamic models, IEEE Transactions on Computer Aided Design of Integrated Circuits and Systems 13 (1994) 702-711

[16] Y. Shi, G. Niu. 2-D analysis of device parasitics for $800 / 1000 \mathrm{GHz} f_{\mathrm{T}} / f_{\mathrm{MAX}} \mathrm{SiGe} \mathrm{HBT}$, In Proc. of the Bipolar/BiCMOS Circuits and Technology Meeting (2005) 252-255

[17] F. Schaeffler. High-mobility Si and Ge structures, Journal of semiconductor science and technology 12 (1997) 1515

[18] D. V. Lang, R. People, J. C. Bean, A. M. Sergent. Measurement of the band gap of $\mathrm{Ge}_{x} \mathrm{Si}_{1-x} / \mathrm{Si}$ strained layer hetero-structures, Applied Physics Letters 47 (1985) 1333

[19] P. Ashburn. SiGe Hetero-junction Bipolar Transistors, John Wiley and sons, Chichetser, (2003) 